\title{
Mobile charging and data gathering in multiple sink wireless sensor networks: how and why
}

\begin{abstract}
Recently, the problem of using efficient the number of mobile devices starting from multisink to go to charge and collect data of sensors such that sensors can work forever has received a great deal of attention in Wireless Rechargeable Sensor Network (WRSN). Many methods have been proposed for the WRSN systems such that mobile device can charge and collect data from sensors. However, most of previous works often require lots of mobile devices while the cost of mobile device is very high. In this paper, we investigate the Periodic Energy Replenishment and Data Collection with multiple sink (PERDCMS) problem and propose a new algorithm, called the Mobile Device Scheduling Algorithm (MDSA), to using limited number of mobile devices for charging and collecting data for sensors. Simulation results show that the MDSA has better performance than other method.
\end{abstract}

Keyword: Wireless rechargeable sensor network; Energy charging; Data collecting; Multiple-sink 